\title{
Two-Dimensional Process Simulation Using Verified Phenomenological Models
}

\author{
Richard B. Fair, Fellow, IEEE, Carl L. Gardner, Michael J. Johnson, Member, IEEE, \\ Stephen W. Kenkel, Member, IEEE, Donald J. Rose, Member, IEEE, \\ John E. Rose, and Ravi Subrahmanyan
}

\begin{abstract}
Two-dimensional (2-D) effects are becoming increasingly important in the diffusion of impurities in submicrometer silicon devices. Existing process simulators cannot accurately model some of these effects. We describe a 2 -D process simulator PREDICT2 that handles implant damage effects, annealing, and lateral diffusion.

PREDICT2 simulates the diffusion of impurities in silicon by using phenomenological diffusion coefficients. The phenomenological models are verified by comparing simulated and experimental results. This approach is contrasted with that of point defect-based simulators.

The experimental technique for measuring impurity profiles in two dimensions is outlined. The method for generating diffusion models is then illustrated by examining 2-D phosphorus diffusion. Numerical simulations and experimental measurements are compared. The numerical methods used in the simulator are described, and directions for future work suggested.
\end{abstract}

\section{Process Simulation Approaches}

\section{A. Introduction}

7 THE DIFFUSION of impurity atoms in silicon is governed by the concentrations of point defects (vacancies and self-interstitials), the diffusion mechanisms, and the boundary conditions of the silicon substrate. For years process simulators have been based on solutions to Fick's second law with time-averaged effective diffusion coefficients. More recently, our understanding of diffusion mechanisms and the importance of point defects has increased. Experiments have been performed to measure point defect diffusion fluxes and recombination and generation characteristics. As a result, some success has been

Manuscript received December 12, 1989; revised March 23, 1990. The work of C. L. Gardner was supported in part by the Microelectronics Center of North Carolina and by the National Science Foundation under Grants DMS-8905872 and DMS-8804592. The work of D. J. Rose was supported in part by the Microelectronics Center of North Carolina and by the Office of Naval Research under Contract N00014-85-K-0487. This paper was recommended by Associate Editor J. G. Fossum.

R. B. Fair is with Design Research and Technology, Microelectronics Center of North Carolina, Research Triangle Park, NC 27709 and the Department of Electrical Engineering, Duke University, Durham, NC 27706.

C. L. Gardner and D. J. Rose are with the Computer Science Department, Duke University, Durham, NC 27706.

M. J. Johnson is with the IBM Corporation, Lexington, KY 40511.

S. W. Kenkel is with the Simulation and Modeling Division, Microelectronics Center of North Carolina, Research Triangle Park, NC 27709.

J. E. Rose and R. Subrahmanyan are with the Microelectronics Center of North Carolina, Research Triangle Park. NC 27709.

IEEE Log Number 9042649 achieved in calculating one-dimensional (1-D) profiles using point defect-based simulators, especially for deep junctions [1]-[3]. However, for modern technologies in which two-dimensional (2-D) simulation is required, low thermal budget processes produce nonequilibrium fluxes of point defects which dominate dopant diffusion and which are extremely difficult to calculate. Thus the very problems which point defect-based simulations are required to solve have been made highly complex by modern processing conditions [4]. We are forced to make some hard choices: 1) will the demand for accurate 2-D simulation wait while we develop more and more complex models for point defect based simulators? 2) In view of point defect modeling uncertainties, are 2-D simulations based on effective diffusion coefficients more accurate?

We recommend "mixing and matching" phenomenological and point defect simulations. By using phenomenological models, our simulator PREDICT2 is not limited by the short diffusion time scales for point defects (the numerical problem for point defects plus impurities is much stiffer than for impurities alone). In addition, PREDICT2 can simulate effects (e.g., the influence of implant damage on diffusion) which are difficult or impossible to model with point defect-based simulators. On the other hand, for certain problems, a more fundamental approach is appropriate. For example, oxidation enhanced diffusion varies along the silicon-oxide boundary as the silicon crystalline orientation changes. This type of problem is ideally suited for rigorous 2-D modeling using a point defect-based program.

\section{B. The Case for the Point Defect-Based Simulators}

The drive to small device geometries has made it necessary to understand diffusion, both laterally and vertically. Griffin and Plummer [5] have pointed out that models based on point defect mechanisms require knowledge of local point defect concentrations with appropriate time constants to describe defect kinetics vertically and laterally. A third time constant is required to describe the time evolution of the 2-D profile as it approaches steady state. Other point defect parameters that are required include diffusivity, generation rates of various sources, recombination or trapping rates of sinks, and point defect concentration gradients [6]. The boundary conditions for 
point defects at the surface are also required. It seems likely that some of these parameters depend on individual wafer or ingot properties. It has already been noted that differences in self-interstitial diffusivities [6]-[12], shown in Fig. 1, may be due to the kind of silicon material: epi, float-zone, or Czochralski [13]. In addition, each process will have to be characterized for its point defect generation or recombination impact.

Other effects that must be addressed include [14], [15] the following.

- Point defect diffusivity is much greater than dopant diffusivity, causing the numerical simulations to be stiff.

- Spatial variation of point defects is important.

- Steady-state distributions cannot be assumed.

- Both the vacancy and self-interstitial profiles must be calculated from their respective continuity equations.

In spite of these obstacles, point defect-based simulation has advantages in simulating complex 2-D structures. Nevertheless, process designers are looking for some relief from the CPU-intensive calculations that are required to simulate even a single process step.

\section{The Challenge}

Until processes and silicon wafers become fully characterized and stable with respect to their point defect parameters, simulators based on solving coupled point defect and dopant continuity equations will be, at best, qualitative. The difficulty in building quantitative simulation models is highlighted by considering process-induced damage by ion implantation. The types of damage produced by implanting depends on ion species, dose, energy, wafer temperature, and orientation. The damage types include point defect clusters, amorphous layers, projected range dislocations, and end-of-range dislocations. Recent work by Orlowski [6] illustrates the role of implantation damage on $\mathrm{P}$ and $\mathrm{B}$ diffusion simulations.

As an implanted wafer is heated, each class of damage will begin to shrink or grow, depending on the annealing ambient. There are no known models available to calculate the point defect generation and recombination characteristics of these damaged regions. Also, the annealing of end-of-range dislocations, for example, depends on the distance from the surface. These effects are quite complex and the physical processes, let alone the mathematics, are not fully understood. Unknown physical processes, such as these, are more easily simulated by modeling their effects on the diffusion of dopants.

\section{The Case for the Phenomenological Simulator}

The phenomenological simulator is based upon empirical models with effective diffusion coefficients of dopants, rather than simulation of point defect concentrations. Examples of such programs include SUPREM-III [16], PREDICT1 [17], and COMPOSITE [18]. Model-based simulation requires calibration with experimental measurements: dopant profiles, oxide thickness, etc. Thus individual models must be created, verified

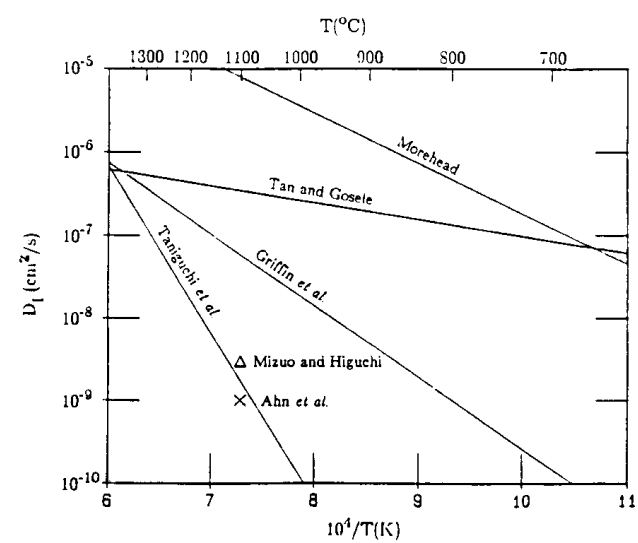

Fig. 1. Current estimates of self-interstitial diffusivity.

against experimental data, and then combined in some manner to account for the integration of process steps in a real technology. Integration methods include "open architecture" approaches which allow the user to insert models into the simulator, and the tight-coupled model approach based on a decision tree architecture [19]. The latter method recognizes that universal models which simulate all conditions are not yet a reality and that a decision tree allows models to be used which are only valid over certain ranges of processing parameters. Also, separating models as tree branches allows for their verification independently from other models. Process integration is accomplished by having small granularity in the branches and by setting flags after each model is executed. The flags describe the condition of the silicon substrate or deposited film sequence, and these then become inputs into the next model selected.

The advantage of phenomenological simulators is that they are pragmatic. Many effects which are of great importance in VLSI processing technology can most easily be modeled in terms of effective parameters with a physical basis. Recent advances in profiling techniques make it feasible to extend effective parameter models to include 2 -D effects. The disadvantage of this type of simulator is that it cannot be used to discover new processes beyond the parameter set of the models. In many ways phenomenological simulation is done in hindsight. The method is to capture data and knowledge in software so that process designers and engineers can be less concerned with known things. To do this successfully requires many experiments to create the data, extensive analysis with sufficient data to make valid models, followed by continued verification of modeling software with each addition to the data set. This process is illustrated below for PREDICT2.

\section{PREDICT2 Simulator}

\section{A. Measurement Techniques}

In order to develop and verify the 2-D PREDICT models, accurate 2-D impurity profiles must be measured. 
The main difficulty involved in these experiments is the determination of lateral diffusion. Several methods for measuring lateral diffusion have been described in the literature. Sheng and Marcus [20] used transmission electron microscopy (TEM) for measuring shallow junctions, including lateral diffusion. Tseng and Wilkins [21] described the measurement of effective channel lengths of MOSFET's by staining cleaved cross sections and measuring the junction with a scanning electron microscope (SEM). Electrical methods, such as the measurement of overlap capacitance, have also been used for indirect calculations of 2-D impurity profiles [22]. Each of these methods has disadvantages: the TEM method uses complex preparation and etching techniques; the cleaving-SEM method does not provide very good resolution; and the electrical methods usually only give reliable information about parameters at the surface.

The method described here is based on the angle lapping and staining technique [23]. Chemical junction staining has been verified extensively for the 1-D case [24]. The profiles are mechanically magnified by cutting the wafers twice, once to enhance the vertical profile and once to magnify the lateral profile. The cut wafers are then stained and the isoconcentration contours are measured by microscopy. Finally, the 2-D profiles are reconstructed from the measured stained contours, using simple scaling and rotation transformations. The contours for different concentrations are measured by repeating the measurements using substrates with different resistivities. This technique has an absolute error resolution of $<0.1 \mu \mathrm{m}$ (smaller features can be resolved), which is sufficient to allow calibration of the simulator. This technique is the best available method for obtaining 2-D profiles.

\section{B. Implant Damage Distributions}

The steps which are important in fabricating submicrometer devices include low thermal budget processing, Ge or Si pre-amorphization implants, ultra-low energy B, $\mathrm{P}$, and As implants, and thin oxides. The physical models needed to simulate these process steps are not simple extrapolations of existing models. For example, during low temperature furnace anneals or high temperature rapid thermal anneals (RTA's), implant damage can anneal or form new, more stable damage arrays, generating excess point defect of both self-interstitial and vacancy types. The excess point defects can either enhance or retard dopant diffusion, depending on the diffusion mechanism. These effects are poorly understood, but they tend to dominate the diffusion process. In addition, surface effects, such as the excess point defects produced during oxidation, are also more important in shallow junction processes.

Fig. 2 is a TEM micrograph showing end-of-range implantation damage, and illustrating that annealing out of ion implantation damage depends on damage depth, annealing ambient, and other factors [25].

Fig. 3 shows simulated profiles with, and without, damage effects. (Fig. 3 uses the same P model as Figs. 6

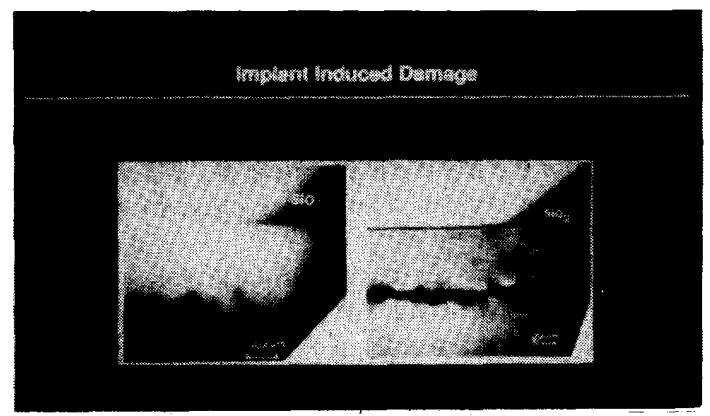

Fig. 2. A TEM micrograph illustrating the influence of the ambient on defect density following 450-deg 10-s anneals in argon and oxygen, respectively.

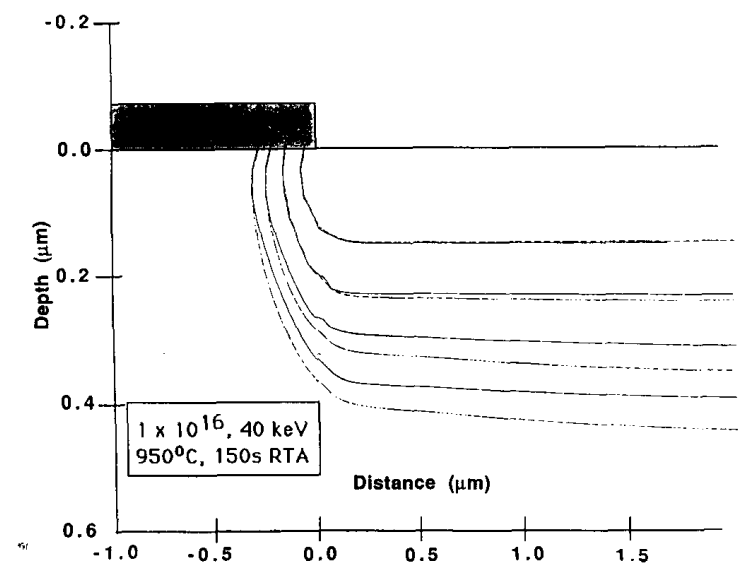

Fig. 3. Simulated profiles for a $10^{16}-\mathrm{cm}^{-2} 40-\mathrm{keV}$ phosphorus implant, diffused for $2.5 \mathrm{~min}$ at $950 \mathrm{deg}$. The deeper curves are the contours where the damage models have been "turned off." The contours are $10^{20}, 10^{19}$, $10^{18}$, and $10^{17} \mathrm{~cm}^{-3}$.

and 7.) The phosphorus implantation used in these simulations leads to the formation of a shallow amorphous layer which causes retarded diffusion of the phosphorus. Substantial damage-related differences are seen in the depth direction. Smaller, but still noticeable, differences appear in the deeper portions of the lateral direction. The agreement in the lateral direction near the surface is due to the absence of amorphous silicon under the masking oxide.

These phenomena are quite complex and, as yet, the physical processes involved are not fully understood. However, recent results suggest that the following qualitative model describes damage annealing and point defect generation for amorphizing implants:

- recovery of the amorphous silicon to single crystal with simultaneous formation of a buried layer of interstitial, perfect dislocation loops [26];

- dislocation loop growth through coalescence by dislocation-dislocation reactions and nonconservative climb processes which generate point defects [27]; 
- at a critical size these loops glide to the Si surface under an attractive force, which ends the point defect generation [28].

Loops must be located within twice their diameter from the surface for the interaction force to be sufficient to pull them out of the crystal. Thus loops close to the surface will disappear first.

To model the enhanced diffusion of high dose implants during RTA or low temperature processing, a point defect-based simulator would need to model all the point defect generation and recombination events. In addition, the distribution of the loops remaining after anneal would have to be known.

As an example, consider the partially annealed structure shown in Fig. 4(a). It is assumed that a high dose implant of $\mathrm{P}$ created an amorphous layer. Subsequent annealing at $800^{\circ} \mathrm{C}$ diffuses the $\mathrm{P}$ and leaves the end-ofrange damage as shown. This damage can play an important role in subsequent processing as well. If an oxidation step is now performed, the damage will shield the underlying $P$ from self-interstitials generated by the oxidizing surface. Oxidation-enhanced diffusion will occur where the damage has disappeared, and the existing loops will grow larger. The result is shown qualitatively in Fig. 4(b). The effect of self-interstitial screening will end when the condition for loop removal is favorable, or the loops become consumed by the oxidation of $\mathrm{Si}$.

The effect of end-of-range damage on $\mathrm{P}$ diffusion wet $\mathrm{O}_{2}$ oxidation is dramatic and is shown in Fig. 5. A $10^{14}-\mathrm{cm}^{-2}, 40-\mathrm{keV} \mathrm{P}$ implant was performed into $117 \AA$ of oxide on crystalline $\mathrm{Si}$ and also into $\mathrm{Si}$ preamorphized by a $10^{15}-\mathrm{cm}^{-2} 50-\mathrm{keV} \mathrm{Si}^{+}$implant. The end-of-range damage is initially at $1000 \AA$ from the surface. After oxidation, little $\mathrm{P}$ diffusion has occurred except for $\mathrm{P}$ gettering to the damage sites. For the sample with no preamorphization, appreciable diffusion has occurred. Accurate, first principal modeling of these effects is beyond current capabilities. Work on handling this problem on a phenomenological basis is described in the following.

\section{Diffusion Models}

The diffusion models are derived from the 1-D models in PREDICT1, which have been verified by comparison with numerous experiments [29]. We are extending this approach to two dimensions. First, physical models are based on experimental measurements, then mathematical models are developed and implemented in the simulator. The parameters in the models are adjusted until the simulation reproduces the measured diffusion profiles. This is done for each set of experimental conditions. A decision tree architecture is used to store parameter values for various conditions [19]. In this way, dozens of models over hundreds of sets of conditions can easily be managed.

The first 2-D models we studied are the phosphorus models, extending the Fair-Tsai model [30] in a manner similar to Lau and Gösele [22]. Their extension of the

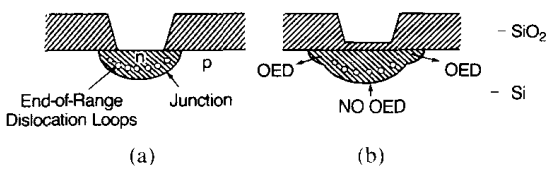

Fig. 4. Effect of end-of-range damage on oxidation-enhanced diffusion of dopants in a 2-D structure (a) p-n junction profile and damage after implant and anneal. (b) Subsequent low T oxidation.

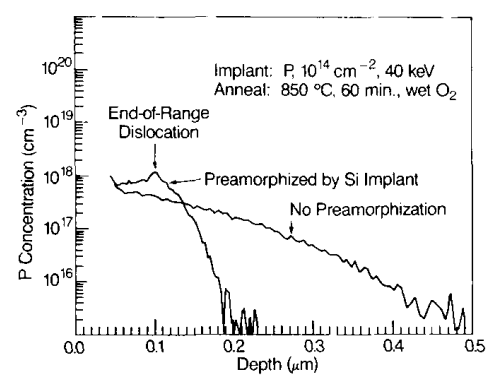

Fig. 5. Effect of end-of-range dislocations on $\mathbf{P}$ diffusion during oxidation.

Hamasaki model to account for 2-D effects has been used with a slight modification. This 2-D extension of the 1-D PREDICT models is adequate in modeling the lateral effects near the surface, but does not agree with experiment in the lateral direction farther from the surface. We are continuing to improve our models to handle these effects.

The Fair-Tsai model accounts for the effects of excess vacancies on the effective diffusivity of phosphorus. The profile of phosphorus concentration in silicon can be divided into surface, kink, and tail regions [31]. The surface region concentration is relatively "flat"; in the kink region, the diffusivity is inversely proportional to the square of the concentration; and in the tail region, the diffusivity is independent of concentration.

It is, of course, impossible to calculate an exact analytical expression for the excess vacancy factor under arbitrary conditions. Instead, we look for an approximate form for this function by considering the steady-state solution of the continuity equations for point defects in a simple situation. Hamasaki [32] has given a solution which assumed a source of self-interstitials at the line $(y$ $=0, x>0)$, corresponding to the free silicon surface, and a perfect sink at the line $(y=0, x>0)$, corresponding to the silicon oxide. Following Lau and Gösele, we have modified this geometry so that the source of point defects is the locus of points $\left\{\left(x_{\varphi}, y_{c}\right): C\left(x_{c}, y_{c}\right)=n_{c}\right\}$, where the point defect-impurity clusters disassociate. (The critical concentration $n_{e}$ is the concentration at which point defects disassociate.) In addition, we have assumed that the Hamasaki factor describes the vacancy distribution. The oxide surface is still assumed to act as a sink. This leads to a set of "scaled" coordinates

$$
u=\frac{\left(x-x_{u^{\prime}}\right)}{L}, \iota^{\prime}=\frac{\left(y-y^{\prime}\right)}{L}, r=\sqrt{u^{2}+u^{2}}
$$


where $L$ is an effective recombination length, taken here to be $25 \mu \mathrm{m}$. Hamasaki's solution gives the concentration of interstitials, normalized by the value at the oxidized surface, as a function $H(u, v)$. In the Fair-Tsai model the effective diffusivity in the tail region, for example, is given by $D_{\text {tail }}=D_{i}^{x}+D_{-}$, where the second term is directly proportional to the concentration of vacancies. Here the $D_{i}^{x}$ term models effects due to neutral point defects,

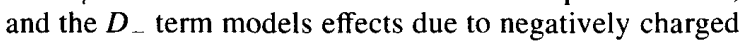
point defects. Using the $H$ factor to calculate this concentration, we thus have 2-D extension of the Fair-Tsai diffusion model. Lau and Gösele have also applied the Hamasaki solution to the Fair-Tsai model, but they did not consider the "kink" region. While the $H$ factor has a fairly complicated form (the reader is referred to [32] for a plot of $H$ as a function of position), qualitatively, this factor leads to different effective diffusion lengths in the lateral and depth direction, without introducing such lengths artificially.

\section{2-D Experiment Versus Simulation}

It has been noted that existing simulators have difficulty in calculating 2-D P diffusion profiles in the vicinity of the edge of an oxide window [4], [23]. A comparison between experimental data and PREDICT2 simulation is shown in Figs. 6 and 7. The implant was $P$ at $10^{15} \mathrm{~cm}^{-2}$ $40 \mathrm{keV}$ made into a wafer with patterned oxide windows with nearly vertical mask edges in $1.4-\mu \mathrm{m}$ thick oxide.

Fig. 6 compares a PREDICT2 RTA (2.5 min at 1050 deg) simulation with experimental data. The discrepancy at the "corner" of the profile is the first sign of a "window" effect. A longer anneal with the same implant (see Fig. 7) accentuates this effect.

In Fig. 7, the samples were annealed in dry $\mathrm{N}_{2}$ for 30 min at $1050^{\circ} \mathrm{C}$, and isoconcentration contours were obtained at $2 \times 10^{14} \mathrm{~cm}^{-3}, 2 \times 10^{15} \mathrm{~cm}^{-3}$, and $2 \times 10^{17}$ $\mathrm{cm}^{-3}$ [23]. Comparing simulated and measured contours at $2 \times 10^{15} \mathrm{~cm}^{-3}$ shows the $P$ diffusion is reduced in the direction of the corner of the profile.

For the simulations the as-implanted profile was approximated by a Pearson-IV distribution in the depth coordinate $(y)$ and a Gaussian in the lateral direction $(x)$. The lateral Gaussian parameters are chosen as in [33]. The parameters can be measured, or obtained from Monte Carlo calculations. The Pearson-IV parameters are adjusted to give good agreement with the 1-D ion implantation models in PREDICT1.

Experimentally, the maximum error near the surfaces is about $0.1 \mu \mathrm{m}$, and near the middle of the oxide window about $0.05 \mu \mathrm{m}$, but the difference between the experimental and simulated results of about $0.3 \mu \mathrm{m}$ at the corner points out the seriousness of the discrepancy. The discrepancy is substantially greater than the estimated uncertainty in the measurements. The long anneal should remove any damage caused by the implant, and the effects of the initial damage will be very small after $30 \mathrm{~min}$, so this "flattening" of the corners is probably not a damage

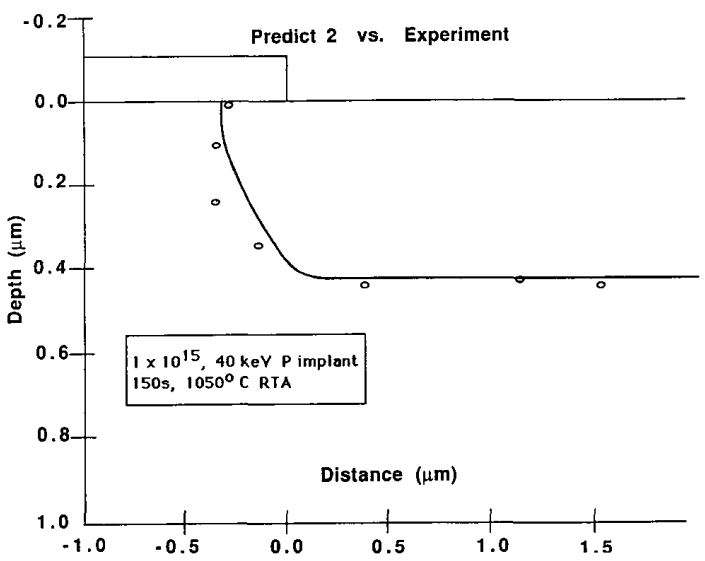

Fig. 6. 2.5-min RTA at $1050 \mathrm{deg} 10^{15}-\mathrm{cm}^{-2} 40-\mathrm{keV}$ phosphorus implant. The contours shown are $2 \times 10^{15} \mathrm{~cm}^{-3}$. The circles are the experimental points, and the curve is the simulation.

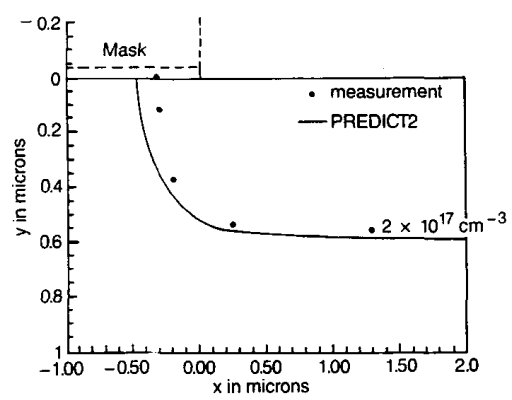

Fig. 7. Simulated and measured $P$ isoconcentration profiles-PREDICT2: $10^{15}-\mathrm{cm}^{-2} 40-\mathrm{keV} P$ implant, diffused for $30 \mathrm{~min}$ at $1050 \mathrm{deg}$. The contours are $2 \times 10^{17} \mathrm{~cm}^{-3}$.

effect. There are two potential sources of error in the implant model: the difference between the 1-D profiles, and the use of a Gaussian distribution in the lateral direction. Some work [34] has shown that the Pearson-IV-Gaussian hybrid may not be a good approximation. The size of this lateral effect, however, is too large to be due solely to the uncertainty in the implantation model.

As an alternative approach, we used SUPREM-IV [35] to model vacancy injection at the oxide mask edge due to decomposition of $\mathrm{SiO}_{2}$ at high temperature [35]. Results are shown in Fig. 8. The fit is good for the $2 \times 10^{17} \mathrm{~cm}^{-3}$ contour as seen in Fig. 8(a). For the $2 \times 10^{14} \mathrm{~cm}^{-3}$ contour in Fig. 8(b), the fit is good near the surface and the mask edge, but is off by about $20 \%$ in the region under the window. This discrepancy may be because 1) the initial simulated implant profile was not deep enough at $2 \times$ $10^{14} \mathrm{~cm}^{-3}$, and 2) the bulk recombination factor was not high enough, causing the effect of the injected vacancies to be too high at that depth, reducing the diffusion of the dopant too much. It is believed that the error due to the implant is less than $10 \%$. Work is in progress to attempt to correct for an error due to excess vacancies in the bulk 


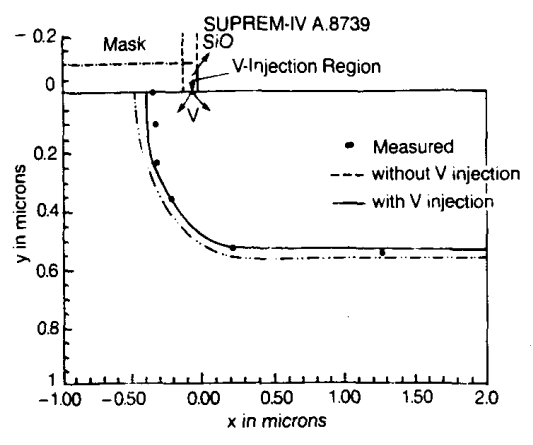

(a)

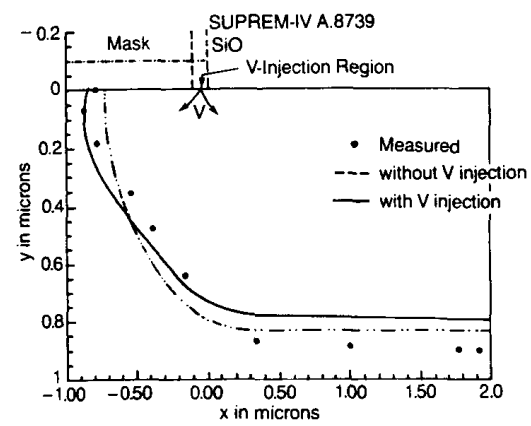

(b)

Fig. 8. Simulated and measured $P$ isoconcentration profiles-SUPREMIV

by increasing the value of the bulk recombination rate. However, the problem of fitting two sets of data when there are several variables is very difficult, because any adjustment of parameters must still satisfy the criterion of physical correctness, which in this case means that the entire set of parameters must be consistent, and allow the point defect populations to come to equilibrium. An inconsistent set of parameters causes the simulator not to converge to a solution. (Further details are provided in [36]).

What is clear from this example is that current 2-D simulators are not adequate for simulating this single-step diffusion problem, regardless of the nature of the simulator. Several potential causes such as window-edge stress have been investigated using PREDICT2, but no satisfactory results were obtained [37]. Significant research and development will be required to solve this problem for either type of simulator.

\section{E. Numerical Methods}

In this section we describe the numerical methods used in the PREDICT2 simulations.

The nonlinear diffusion process is described by a set of conservation laws for impurities

$$
\frac{\partial C_{i}}{\partial t}=\nabla \cdot\left(\sum_{j} D_{i j} \nabla C_{j}\right) \equiv F_{i}
$$

where $C$ is the impurity concentration in atoms per cubic centimeter, $D$ is the phenomenological diffusion coefficient in square centimeters per second, and $i, j=1, \cdots$, $N$ label the types of impurities. Note that $D=D\left(C_{1}, \cdots\right.$, $C_{N}$ ) includes the effects of the coupling of the impurity ions to the electric field.

We use the composite TRBDF2 method [38] to step in time. To integrate (1) from $t=t_{n}$ to $t_{n+1}=t_{n}+\Delta t_{n}$, we apply the trapezoidal rule (TR) to advance the solution from $t_{n}$ to $t_{n+\gamma}=t_{n}+\gamma \Delta t_{n}$ :

$$
C^{n+\gamma}-\gamma \frac{\Delta t_{n}}{2} F^{n+\gamma}=C^{n}+\gamma \frac{\Delta t_{n}}{2} F^{n}
$$

and then use the second-order backward differentiation formula (BDF2) to advance the solution from $t_{n+\gamma}$ to $t_{n+1}$ :

$$
\begin{aligned}
C^{n+1} & -\frac{1-\gamma}{2-\gamma} \Delta t_{n} F^{n+1} \\
= & \frac{1}{\gamma(2-\gamma)} C^{n+\gamma}-\frac{(1-\gamma)^{2}}{\gamma(2-\gamma)} C^{n} .
\end{aligned}
$$

This composite one-step method is second-order accurate and L-stable (see [38] for the definitions of A-stable and L-stable).

The importance of L-stability for diffusion is illustrated for a 1-D computation in Fig. 9. After a single timestep with $\Delta t=50 \Delta t_{\text {Euler }}=50 \Delta y^{2} / 2 D_{\max }$ the TR method, which is A-stable but not L-stable, exhibits severe unphysical oscillations near the maximum of $C$.

We linearize $F^{n+1}$ in (3) (and similarly $F^{n+\gamma}$ in (2)) by approximating

$$
F_{(k+1)}^{n+1}=F_{(k)}^{n+1}+\left(\frac{\delta F}{\delta C}\right)_{(k)}^{n+1} \delta C_{(k)}^{n+1}
$$

where $k=0,1, \cdots$ labels the Newton iterations, and the Fréchet derivative

$$
\frac{\delta F}{\delta C} *=\nabla \cdot\left(\frac{\delta D}{\delta C} * \nabla C\right)+\nabla \cdot(D \nabla *) .
$$

The new solution is obtained by setting

$$
C_{(k+1)}^{n+1}=C_{(k)}^{n+1}+\lambda \delta C_{(k)}^{n+1}, \quad C_{(0)}^{n+1}=C^{n+\gamma}
$$

where $\lambda$ is a damping factor [39] between 0 and 1 , chosen to insure that the norm of the residual for (2) or (3) decreases monotonically. At each TR or BDF2 partial step, we iterate until the Newton method converges.

The Newton equation for the TR partial step is

$$
\begin{aligned}
& {\left[1-\gamma \frac{\Delta t_{n}}{2}\left(\frac{\delta F}{\delta C}\right)_{(k)}^{n+\gamma}\right] \delta C_{(k)}^{n+\gamma}} \\
& \quad=-\left(C_{(k)}^{n+\gamma}-C^{n}\right)+\gamma \frac{\Delta t_{n}}{2}\left(F_{(k)}^{n+\gamma}+F^{n}\right) \equiv-G_{\mathrm{TR}}
\end{aligned}
$$

where $G_{\mathrm{TR}}$ is the residual for (2). 


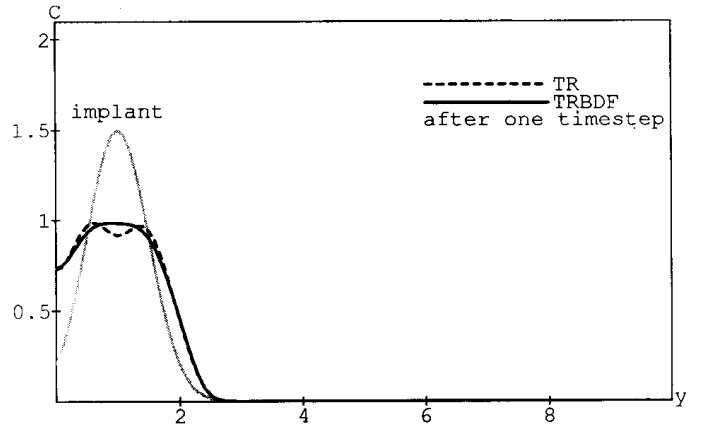

Fig. 9. TR versus TRBDF for a single timestep with $\Delta t=50 \Delta t_{\text {Euler. }} C$ is in $10^{20} \mathrm{~cm}^{-3}$ and $y$ is in $0.1 \mu \mathrm{m}$.

The Newton equation for the BDF2 partial step is

$$
\begin{aligned}
{[1-} & \left.\frac{1-\gamma}{2-\gamma} \Delta t_{n}\left(\frac{\delta F}{\delta C}\right)_{(k)}^{n+1}\right] \delta C_{(k)}^{n+1} \\
= & -\left[C_{(k)}^{n+1}-\frac{1}{\gamma(2-\gamma)} C^{n+\gamma}+\frac{(1-\gamma)^{2}}{\gamma(2-\gamma)} C^{n}\right] \\
& +\frac{1-\gamma}{2-\gamma} \Delta t_{n} F_{(k)}^{n+1} \equiv G_{\mathrm{BDF} 2}
\end{aligned}
$$

where $G_{\mathrm{BDF} 2}$ is the residual for (3).

Note that the Jacobians for the TR and BDF2 partial steps have the same form if $\gamma=2-\sqrt{2}$. If the solution is varying slowly, then Jacobian factorizations may be reused (Newton-Richardson method) while retaining quadratic convergence of the Newton method [38].

The timestep size $\Delta t$ is adjusted dynamically within a window $\left[\Delta t_{\min }, \Delta t_{\max }\right]$ by monitoring a divided-difference estimate of the local truncation error $\tau$ [38]:

$$
\begin{aligned}
\tau^{n+1} & =k \Delta t_{n}^{3} C^{(3)} \\
& \approx 2 k \Delta t_{n}\left[\frac{1}{\gamma} F^{n}-\frac{1}{\gamma(1-\gamma)} F^{n+\gamma}+\frac{1}{1-\gamma} F^{n+1}\right]
\end{aligned}
$$

where

$$
k=\frac{-3 \gamma^{2}+4 \gamma-2}{12(2-\gamma)}
$$

and $C^{(3)}$ denotes the third time derivative of $C$. Yeager and Dutton [2] use the classic Milne method for selecting $\Delta t$. The is appropriate for very stiff problems (e.g., for point defect-based simulations). Our phenomenological simulations are not nearly as stiff, and we find better performance with the divided difference estimator, especially for the case of diffusion of a single species.

Notice that we linearize (2) and (3) first and then discretize in space. Equations (7) and (8) are discretized by the box method (see, e.g., [40]) using central differences. The resulting discretized linear systems are solved using Bank's sparse matrix package (see, e.g., [38]), or, when

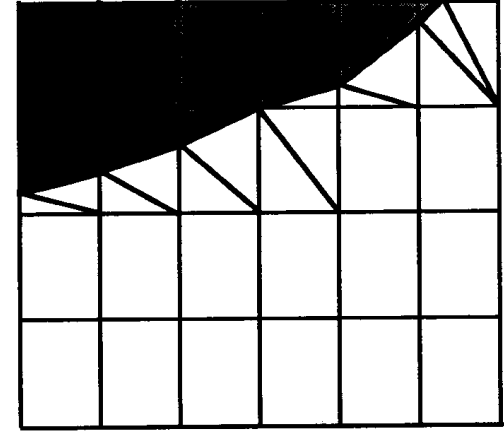

Fig. 10. The finite difference grid near the $\mathrm{Si} / \mathrm{SiO}_{2}$ boundary.

more efficient for problems in rectangular geometries, with a vectorized LINPACK banded solver.

The extension of PREDICT to 2-D is not a simple generalization of the 1-D PREDICT because the moving oxide boundary (the "front"), which in 1-D is a point, has become a curve in 2-D. The curve is directed and is represented as a linked list of straight line elements. Left and right states are stored on the curve, allowing for discontinuous changes in the concentrations across the oxide boundary. We use an underlying finite difference grid of rectangles, plus a set of triangulated rectangles (see Fig. 10) near the front. ${ }^{1}$ An algorithm based on minimizing the occurrence of acute triangles is used to uniquely triangulate a rectangle which is intersected by the front. We do not allow a given rectangle to be intersected more than once by the front.

The curve representing the oxide boundary is currently propagated during a time $\Delta t$ using a phenomenological model. Regridding occurs only in retriangulating around the oxide boundary, a set of $O(N)$ rectangles compared to the computational region of $O\left(N^{2}\right)$ rectangles.

The grid of rectangles plus triangles near the front may be automatically refined in a self-similar fashion. In other words, the regions which are refined have an underlying finite difference grid of rectangles plus triangulated rectangles near the front.

\section{F. Future Work}

The ion implantation and oxidation models in PREDICT1 must be modified to incorporate 2-D effects. Until more data (including channeling effects) are available, the 2-D implantation models will have a Gaussian lateral distribution and use the phenomenological 1-D PREDICT1 modes in the depth direction. The first step in implementing models for oxide growth is similar; we will use an analytical 2-D "bird's beak" model [41] as a first approximation, and then refine the model as experiments

'This method is inspired by the "front-tracking method" of J. Glimm, O. McBryan, et al. See, for example, I. L. Chern, J. Glimm, O. McBryan, B. Plohr, and S. Yaniv, "Front tracking for gas dynamics," J. Comp. Phys., vol. 62, pp. 83-110, 1986. 
indicate. The oxidation model will incorporate the modified 1-D Deal-Grove model that is currently used in PREDICT1.

An environment for developing and maintaining the phenomenological models in PREDICT2 is currently under development [42]. This system allows models to be created by experts in physical modeling with little programming effort. The concerns of the software engineer have been separated from the those of the model developer in order to increase the productivity of each.

Future enhancements of the numerical methods in PREDICT2 will include automatic mesh refinement [43], a waveform Newton timestepping scheme [44], and simulating oxidation using viscoelastic dynamical equations.

\section{REFERENCES}

[1] D. Mathiot and J. C. Pfister, "Dopant diffusion in silicon-a consis tent view involving nonequilibrium defects, "J. Appl. Phys., vol. 55 , pp. $3518-3530,1984$.

[2] H. R. Yeager and R. W. Dutton, "An approach to solving multiparticle diffusion exhibiting nonlinear stiff coupling, "IEEE Trans. Electron Devices, vol. ED-32, pp. 1946-1976, 1985

[3] P. Pichler, W. Jungling, S. Selberherr. E. Guerrero, and H. W. Potzl, "Simulation of critical IC-fabrication steps." IEEE Trans. Computer-Added Design, vol. CAD-4, pp. 384-397, 1985.

[4] R. B. Fair, C. L. Gardner, M. J. Johnson. S. W. Kenkel, D. J. Rose J. E. Rose, and R. Subrahmanyan, Workshop Numerical Modeling of Processes and Devices for Integrated Circuits (NUPAD II), San Diego, May 1988

[5] P. B. Griffin and J. D. Plummer, "Process physics determining 2D impurity profiles in VLSI devices." in IEDM Tech. Dig., 1986, pp. $522-525$.

[6] M. Orlowski, "Unified model for impurity diffusion in silicon," Appl. Phys. Lett., vol. 53, pp. 1323-1325, 1988.

[7] K. Taniguchi, D. A. Antoniadis, and Y. Matsushita, "Kinetics of self-interstitials generated at the $\mathrm{Si} / \mathrm{SiO} 2$ interface, "Appl. Phys. Lett. . vol. 42, pp. 961-963, 1983.

[8] S. Mizuo and H. Higuchi, "Effects of back-side oxidation on B and P diffusion in Si directly masked with Si3N4 films. " J. Electrochem. Soc., vol. 129, pp. 2292-2295, 1982.

[9] S. T. Ahn, P. B. Griffin, J. D. Shott, J. D. Plummer, and W. A Tiller, "A study of silicon interstitial kinetics using silicon membranes-applications to 2D dopant diffusion." J. Appl. Phys., vol 62, pp. $4745-4755,1987$.

[10] T. Y. Tan and U. Gösele. "Point-defects, diffusion-procsses, and swirl defect formation in silicon." Appl. Phys., vol. A37, pp. 1-17, 1985.

[11] P. B. Griffin, P. M. Fahey, J. D. Plummer, and R. W. Dutton, "Measurement of silicon interstitial diffusivity," Appl. Phys. Lett. vol. 47, pp. 319-321, 1985

[12] F. F. Morehead. "The diversity of self-interstitials in silicon," in Mat. Res. Soc. Symp. Proc, vol. 104. 1988. pp. 99-104.

[13] P. M. Fahey, P. B. Griffin, and J. D. Plummer, "Point defects and dopant diffusion in silicon," Rev. Modern Phys., vol. 61, pp. 289234,1989

[14] M. R. Kump and R. W. Dutton. "The efficient simulation of coupled point-defect and impurity diffusion," IEEE Trans. Computer-Aided Design, vol. 7, pp. 191-204, 1988

[15] M. E. Law and R. W. Dutton, "Verification of analytic point-defect models using SUPREM-IV," IEEE Trans. Computer-Aided Design, vol. 7.pp. 181-190, 1988

[16] D. A. Antoniadis and R. W. Dutton, " Models for computer-simulation of complete IC fabrication process," IEEE Trans. Electron De vices, vol. ED-26. pp. 490-500. 1979.

[17] R. B. Fair, "Low thermal-budget process modeling with the PREDICT computer-program," IEEE Trans. Electron Devices, vol. 35 pp. $285-293,1988$

[18] J. Lorenz, J. Pelka, H. Ryssel, A. Sacks, A. Sadl, and M. Svoboda
"Composite-A complete modeling program of silicon technology," IEEE Trans. Electron Devices, vol. ED-32, pp. 1977-1986, 1985

[19] R. B. Fair and J. E. Rose, "A deep decision tree approach to modeling submicron silicon technologies, "in ICCAD Dig. Tech. Papers ICCAD-87, 1987, pp. 248-251.

[20] T. T. Sheng and R. B. Marcus, "Delineation of shallow junctions in silicon by transmission electron microscopy," J. Electrochem. Soc., vol. $128, \mathrm{pp} .881-884,1981$.

[21] W. F. Tseng and B. R. Wilkins, "Direct observations of n-MOSFET channel lengths," J. Electrochem. Soc., vol. 134, pp. 1258-1260. 1987.

[22] F. Lau and U. Gösele, "Two-dimensional phosphorus diffusion for soft drains in silicon MOS transistors," Appl. Phys.. vol. A40, p. 101, 1986.

[23] D. R. Subrahmanyan, H. Z. Massoud, and R. B. Fair. "Experimental characterization of two-dimensional dopant profiles in silicon using chemical staining," Appl. Phys. Lett., vol. 52, pp. 2145-2147, 1988.

[24] R. Subrahmanyan, H. Z. Massoud, and R. B. Fair, "Accurate determination of junction depths by chemical staining," presented at ASTM Symp. on Semiconductor Characterization, Santa Clara, Feb. 1988.

[25] A. C. Ajmera and G. A. Rozgonyi, "Elimination of end-of-range and mask edge lateral damage in $\mathrm{Ge}+$ preamorphized, B + implanted $\mathrm{Si}$, “ Appl. Phys. Lett., vol. 49, pp. 1269-1271, 1986.

[26] M. Servidori, "Characterization of lattice damage in ion-implanted silicon by multiple crystal X-ray-diffraction," Nucl. Instrum. Methods, vol. B19, pp. 443-449, 1987.

[27] J. D. Verhoeven, Fundamentals of Physical Metallurgy. New York: Wiley, 1975.

[28] J. Narayan and K. Jagannadham, "Effect of free-surface and interface on thermal annealing of dislocation loops in silicon," J. Appl. Phys., vol. 62, pp. 1694-1697, 1987.

[29] R. B. Fair and R. Subrahmanyan, "PREDICT -A new design tool for shallow junction processes," MCNC Tech. Rep. TR85-005. 1985.

[30] R. B. Fair and J. C. C. Tsai, "Profile parameters of implanted-diffused arsenic layers in silicon," J. Electrochem. Soc., vol. 123, pp. 583-586, 1976.

[31] R. B. Fair, Impurity Doping Processes in Silicon, F. F. Y. Wang, ed. Amsterdam, The Netherlands: North-Holland 1981.

[32] M. Hamasaki, "On an analytical solution for two-dimensional diffusion of silicon self-interstitials during oxidation of silicon," SolidState Electron., vol. 25, pp. 1-4, 1982.

[33] H. Ryssel and I. Ruge, Ionenimplantation. Stuttgart, Germany: Teubner, 1978.

[34] G. Hobler, E. Langer, and S. Selberherr, "Two-dimensional modeling of ion implantation." in Proc. Second Int. Conf. on Simulation of Semiconductor Devices and Processes, Swansea, U.K., July 1986. pp. $256-270$.

[35] S. T. Ahn, H. W. Kennel, J. D. Plummer, and W. A. Tiller, "Vacancy generation at the $\mathrm{Si} / \mathrm{SiO} 2$ interface caused by $\mathrm{SiO}$ formation," in Proc. Fall Meeting Electrochemical Society, Chicago, 1988, pp. $407-408$.

[36] R. B. Fair and R. Subrahmanyan, "Phenomenological versus pointdefect-based process modeling: Where should you put your money?." MCNC Tech. Rep. TR89-18, 1989.

[37] R. B. Fair, H. Massoud, and R. Subrahmanyan, "Comparison of measured and simulated two-dimensional phosphorus diffusion profiles in silicon," to be published.

[38] R. E. Bank, W. M. Coughran, W. Fichtner, E. Grosse, D. J. Rose and R. K. Smith, "Transient simulation of silicon devices and circuits," IEEE Trans. Computer-Aided Design, vol. CAD-4, pp. 436$451,1985$.

[39] R. E. Bank and D. J. Rose, "Global approximation Newton methods," Numer. Math., vol. 37. pp. 279-295, 1981

[40] R. E. Bank, D. J. Rose, and W. Fichtner, "Numerical methods for semiconductor device simulation," SIAM J. Sci. Stat. Comput., vol. 4, pp. 416-435, 1983

[41] N. Guillemot, G. Pananakakis, and P. Chenevier, "A new analytica model of the "bird's beak'," IEEE Trans. Electron Devices, vol. ED-34, p. 5, 1987.

[42] J. E. Rose, to be published.

[43] M. J. Berger. "Adaptive finite difference methods in fluid dynamics," to be published.

[44] D. J. Erdman and D. J. Rose. "A Newton waveform relaxation algorithm for circuit simulation," in ICCAD Dig. Tech. Papers ICCAD-89, 1989, pp. 404-407. 


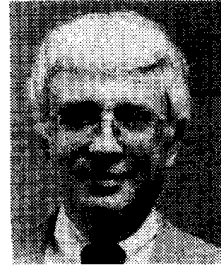

Richard B. Fair (S'63-M'75-SM'75-F'90) received the B.S.E.E. degree from Duke University in 1964, the M.S.E.E. degree from Pennsylvania State University in 1966, and the Ph.D. degree from Duke University in 1969.

In 1969 he joined the Semiconductor Device Laboratory, Bell Laboratories, Reading, PA. As a member of the technical staff, his primary responsibilities were in the areas of processing and modeling of both unipolar and bipolar transistors. In 1973, he became Supervisor of the Semiconductor Device Development Group. He has had responsibility for the development of power transistors, IMPAT diodes, and other microwave devices, as well as MOS technology and IC design. He currently holds appointments as Professor of Electrical Engineering at Duke University and as Vice President of Design Research and Technology at the Microelectronics Center of North Carolina. He has authored over 95 refereed journal articles, seven book chapters and given over 70 invited talks at conferences in the areas of impurity diffusion, ion implantation, and device physics.

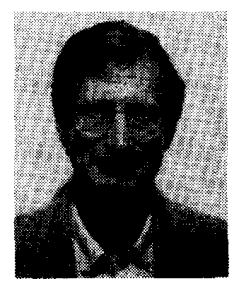

Carl L. Gardner received the B. A. degree from Duke University in 1973 and the Ph.D. degree from MIT in 1981.

$\mathrm{He}$ is currently an Associate Professor of Com puter Science at Duke University. Previously he was an Assistant Research Scientist at the Courant Institute, Assistant Professor of Physics at Bowdoin College, and Instructor of Physics at MIT. His current research interests lie in semiconductor device and process simulations, and computational granular and fluid dynamics.

Dr. Gardner is a member of SIAM and the American Physical Society.

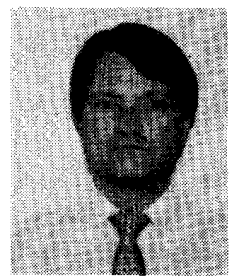

Michael J. Johnson (M'89) received the B.S.E.E. degree from the University of Florida in 1978, the M.S. degree from the University of Kentucky in 1983, and the M.A. degree from Duke University in 1987. He is currently working toward the Ph.D. dgree at Duke University.

He joined IBM in 1978, where he has been involved in the design and analysis of electronic circuits and devices, most recently in the area of power conversion.

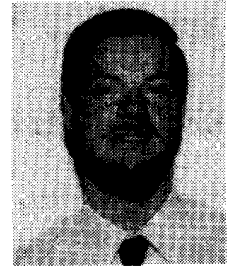

Stephen W. Kenkel (M'86) received the B.S. degree in 1975 and the $\mathrm{Ph} . \mathrm{D}$. degree in 1981 from the University of Kentucky.

From 1981 to 1984 he was a research associate at the University of North Carolina. In 1984 he joined the Microelectronics Center of North Carolina, where he currently manages the Simulation and Modeling group. His interests include process simulation, circuit simulation, scientific computing, and applications of parallel architectures to VLSI technology development and circuit design.

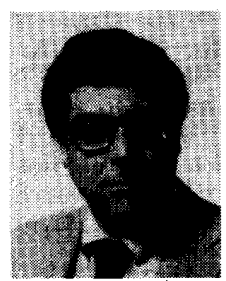

Donald J. Rose ( $\left.\mathrm{M}^{\circ} 89\right)$ received the B.A. degree from the University of California at Berkeley in 1966, and the M.A. and Ph.D. degrees from Harvard University in 1967 and 1970 , respectively.

From 1970 to 1984 , he held research positions at the University of Denver, Harvard University, Vanderbilt University, and AT\&T Bell Laboratories. In 1984, he became Chair of the Computer Science Department at Duke University, where he investigates problems involving numerical analysis and scientific computation.

Dr. Rose is a member of SIAM, AMS, MAA, and ACM.

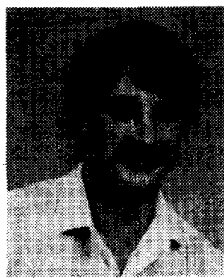

John E. Rose received the B.S. degree from Rensselaer Polytechnic Institute in 1981 and the M.S. degree from North Carolina State University in 1985 . He is currently a graduate student in computer science at Duke University.

He has been employed at the Microelectronics Center of North Carolina since 1986, where he has been working in the field of semiconductor process simulation.

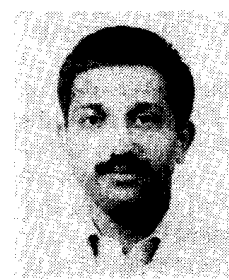

Ravi Subrahmanyan received the B.Tech. degree from the Indian Institute of Technology, Bombay, in 1983 and the M.S. and Ph.D. degrees from Duke University in 1985 and 1988, respectively.

His research concerned the measurement of twodimensional diffusion profiles and the modeling of two-dimensional diffusion in silicon. He was at the Microelectronics Center of North Carolina from 1987 to 1989 , where he worked on process simulation and the circuit simulator CAzM. He is currently with Motorola, Inc., Austin, TX, where he works on process and device simulation. 